\title{
Behçet disease: evaluation of clinical manifestations in Turkish children
}

\author{
Erkan Demirkaya', Celal Saglam*, Turker Turker², Sevcan Bakkaloglu³ , Balahan B Makay ${ }^{4}$, Bora Gulhan ${ }^{5}$, Banu Acar $^{6}$, \\ Nuray A Ayaz ${ }^{7}$, İsmail Dursun ${ }^{8}$, Adem Polat ${ }^{1}$, Cetin Kocabiyik', Faysal Gok ${ }^{1}$, Erbil Unsal ${ }^{4}$, Ozgur Kasapcapur ${ }^{9}$, \\ Seza Özen ${ }^{5}$, for the FMF Arthritis Vasculitis and Orphan Disease Research in Paediatric Rheumatology (FAVOR)
}

From 18th Pediatric Rheumatology European Society (PReS) Congress

Bruges, Belgium. 14-18 September 2011

\section{Objective}

We analyzed the clinical manifestations of Pediatric Behcet disease (PED-BD) in Turkey. We also evaluated the correlation between the physician's global assessment of disease activity (PGA) and Behçet's Syndrome Activity Scale (BSAS) which is one of the activity indices in our cohort.

\section{Method}

8 University hospitals in Turkey enrolled children with a clinical diagnosis of PED-BD. We examined chronologically the onset of individual symptoms in each patient. As an activity index we used BSAS which have been developed to assess the activity specifically for BD in adults. Correlation between the PGA, and BSAS was determined.

\section{Results}

In 54 patients the mean age at the first symptom was $117.50 \pm 45.20$ months. BD was suspected at a mean age of $143.56 \pm 39.63$ months. The mean delay between the first symptom and BD suspicion was 27.36 \pm 27.15 months. The most common manifestations were oral ulcer $96.3 \%(n=52)$, uveitis $46.3 \%(n=25)$, genital ulcer $37 \%(\mathrm{n}=20)$, pustuler lesion $37 \%(\mathrm{n}=20)$, erythema nodosum $24.1 \%(\mathrm{n}=13)$ respectively. Bilateral uveitis was found in $27.8 \%(n=15)$ patients. Pathergy phenomen was positive in $37 \%(\mathrm{n}=20)$ patients. Family history of $\mathrm{BD}$ was present in $38.9 \%(\mathrm{n}=21)$ patients. HLA-B51 carrier rate was $53.7 \%(n=29)$. BSAS was assessed for the 52 patients in our cohort and a moderate correlation between the BSAS and PGA was demonstrated $(r=0.305$, $\mathrm{p}=0.025$ ).

\section{Conclusion}

Our study indicates that BSAS may be a beneficial and practical index to define the disease activity in PED-BD and that further studies with a large cohort are required.

\section{Author details}

${ }^{1}$ Gülhane Military Medical Academy, Pediatric Nephrology and Rheumatology Unit, Ankara, Turkey. ${ }^{2}$ Gulhane Military Medical Faculty, Department of Public Health, Division of Epidemiology, Ankara, Turkey. ${ }^{3}$ Gazi University Medical School, Pediatric Nephrology and Rheumatology Unit, Ankara, Turkey. ${ }^{4}$ Dokuz Eylul University Medical School, Pediatric Immunology and Rheumatology Unit, İzmir, Turkey. ${ }^{5}$ Hacettepe University Medical School, Pediatric Nephrology \& Rheumatology Unit, Ankara, Turkey. ${ }^{6}$ Ministry of Health Diskapi Children's Hospital, Diskapi, Ankara, Turkey. ${ }^{7}$ SB Istanbul Bakırköy Maternity and Childrens Education and Research Hospital, Division of Pediatric Rheumatology, Istanbul, Turkey. ${ }^{8}$ Erciyes University Medical School, Pediatric Nephrology \& Rheumatology Unit, Kayseri, Turkey. ${ }^{9}$ Istanbul University Cerrahpasa Medical School Department of Pediatric Rheumatology, Istanbul, Turkey.

Published: 14 September 2011

\section{doi:10.1186/1546-0096-9-S1-P19}

Cite this article as: Demirkaya et al:: Behçet disease: evaluation of clinical manifestations in Turkish children. Pediatric Rheumatology 20119 (Suppl 1):P19.

\footnotetext{
* Correspondence: dr.csaglam@yahoo.com

${ }^{1}$ Gülhane Military Medical Academy, Pediatric Nephrology and

Rheumatology Unit, Ankara, Turkey

Full list of author information is available at the end of the article
} 